\title{
Tableting process optimisation with the application of fuzzy models
}

\author{
Aleš Belič ${ }^{a}$ Igor Škrjanc ${ }^{a}$ Damjana Zupančič Božič ${ }^{b}$ \\ Franc Vrečer b,c \\ ${ }^{a}$ Faculty of Electrical Engineering, University of Ljubljana, Ljubljana, Tržaška \\ cesta 25, Slovenia \\ ${ }^{\mathrm{b}}$ Krka d.d., Novo mesto, Šmarješka cesta 6, 8501 Novo mesto, Slovenia \\ ${ }^{\mathrm{c}}$ University of Ljubljana, Faculty of Pharmacy, Aškerčeva cesta 7, 1000 Ljubljana, \\ Slovenia
}

\begin{abstract}
A quality-by-design (QbD) principle, including process analytical technology, is becoming the principal idea in drug development and manufacturing. The implementation of $\mathrm{QbD}$ into product development and manufacturing requires larger resources, both human and financial, however, large-scale production can be established in a more cost-effective manner and with improved product quality. The objective of the present work was to study the influence of particle size distribution in powder mixture for tableting, and the settings of the compression parameters on the tablet quality described by the capping coefficient, standard deviations of mass and crushing strength of compressed tablets. Fuzzy models were used for modelling of the effects of the particle size distribution and the tableting machine settings on the tablet quality. The results showed that the application of mathematical models, based on the contemporary routinely-measured quantities, can significantly improve the trial-and-error procedures.
\end{abstract}

Key words: Dry granulation, Tableting, Capping, ANN, Fuzzy models, Mathematical model

Email address: ales.belic@fe.uni-lj.si (Aleš Belič).

$U R L:$ msc.fe.uni-lj.si (Aleš Belič).

Preprint submitted to Int. J. Pharm.

5th January 2010 


\section{Introduction}

The advantages of adopting the quality-by-design (QbD) principle together with process analytical technology (PAT) in drug development and manufacturing are being recognised by the pharmaceutical industry (Frake et al., 1997; Informa, 2007). The implementation of QbD and PAT may be more expensive during product development, however, large-scale production can be established in a more cost-effective manner and with improved product quality. The main advantage of PAT is its flexibility and the ability to adapt to new situations in the process in a short time, since the system can detect production deviations and react by adjusting the process parameters before the deviations can affect the product quality.

Tablets are produced by compressing a powder mixture containing the active ingredient and auxiliary materials into a solid form. The tablet quality can be described by several parameters such as: accurate tablet mass, minimal variations in hardness, capping coefficient, and several others. Capping can occur if the intensity of the elastic relaxation overcomes the strength of the inter-particulate bonding formed during compression, leading to separation of the upper part of the tablet from the tablet body (Picker, 2001).

Many studies on the influence of the powder's mechanical characteristics on the cohesion, the deformation mechanisms, and the elastic recovery of the tablet have been performed in the past (Informa, 2007; Sebhatu et al., 1997; Rios, 2006; Sorensen et al., 2006; Zhang et al., 2003; Gohel and Jogani, 2002; Sucker, 1982; Li et al., 2004; Parrott, 1990; Nyström et al., 1993; Luangtana-Anan and Fell, 1990; Sonnergaard, 2006). Real multicomponent mixtures of ingredients were rarely evaluated in the published studies and practical experiences show that applying models, developed in laboratory-scale studies, i.e.,(Wu et al., 2005; Ilkka and Paronen, 1993; Carstensen, 1996; Busignies et al., 2006) for large-scale production situations often give unsatisfactory results due to the complexity of mixture properties and industrial equipment. The mechanical properties of powder mixtures with a large number of components are too complex to be described in a transparent theoretical mathematical model. The mathematical models developed in several studies (Sebhatu et al., 1997; Rios, 2006; Sorensen et al., 2006; Zhang et al., 2003; Gohel and Jogani, 2002; Sucker, 1982; Li et al., 2004; Parrott, 1990; Nyström et al., 1993; LuangtanaAnan and Fell, 1990; Sonnergaard, 2006) are often not precise enough for the purpose of adjusting the parameters of a tableting machine to inter-batch differences.

The objective of the present work was to study the influence of particle size and the process parameters on the tablet quality, described by capping tendency and standard deviations of mass and crushing strength. Indirectly, the influence of dry granulation process on the quality of the tablets was studied through its influence on particle size distribution. We investigated the use of fuzzy models for the prediction of tablet quality from the routinely mea- 
sured mechanical properties of the powder mixture and the tableting machine's settings. For the model development we used data of tablets produced from powders with different mechanical properties but with the same composition. In our previous research publication (Belič et al., 2009) the prediction of the capping coefficient was studied, while the whole combination of factors, contributing to the tablet quality, was studied in the present work. Model tablets were produced using several different settings of the tableting machine. We also evaluated the efficiency of the fuzzy model for the optimisation of the tableting parameters for the known properties of a powder batch as a basis for controlling the process in the PAT system (Informa, 2007).

\section{Materials and Methods}

The method for optimising the tableting process using fuzzy models was developed and tested on a high-capacity Killian T300/40 rotary press with formulation containing high amount of active ingredient which exhibits poor flow and compressibility characteristics and intensive capping tendency. A dry granulation of this formulation led to a larger particle size, improved powder flowability, better compressibility properties and a significantly lower capping tendency (Zupančič-Božič et al., 2008). Optimisation of tableting setting parameters such as main compression force, pre-compression force and tableting speed can additionally contribute to the quality of the tablets. The data was organised in a matrix where each column represented one measured quantity and each row represented one tablet type. The following quantities were measured for all tablet types: powder distribution over eight particle size ranges $\left(\mathrm{d}_{1}-\mathrm{d}_{8}\right)$, main compression force $(F)$, pre-compression force $(f)$, tableting speed $(v)$, standard deviation of crushing strength $\left(\sigma_{F}\right)$, standard deviation of mass $\left(\sigma_{m}\right)$, and capping coefficient $(\mathrm{CC})$. For the model's evaluation a qualitycorrelation coefficient $\mathrm{R}^{2}$ was used:

$$
R^{2}=1-\frac{\mathrm{SSE}_{M}}{\mathrm{SSE}_{T}}
$$

where $\mathrm{SSE}_{M}$ is the sum of the squared error between the model prediction and the target, and $\mathrm{SSE}_{T}$ is the sum of $\mathrm{SSE}_{M}$ and the average of the target values. The model reliability was estimated as the maximal difference of the calculated and the measured value for each quality factor $\left(\sigma_{F}, \sigma_{m}\right.$, or CC). 


\subsection{Production of Model Tablets}

In order to study the influence of the particle size distribution of powder mixtures on tablet quality, three types of powder mixtures for tableting were prepared:

a) powder mixture for direct tableting (Type: Direct - 1 sample),

b) powder mixture prepared by slugging (dry granulation on a rotary tablet press), using different setting parameters of tableting speed and compression pressure (Type: Slugging - 4 samples - see Table 1),

c) powder mixture prepared by dry granulation on a roller compactor using different parameters of compacting speed and pressure (Type: Roller 4 samples - see Table 1).

[Table 1 about here.]

The qualitative and quantitative compositions of all the powder mixtures were the same: active ingredient (M-112, provided by KRKA, d.d., Novo mesto), 75\% (w/w), microcrystalline cellulose (MCC, Avicel PH 101, FMC, Germany), 15\% (w/w), cation exchange resin - Amberlite IRP88 (Rohm and Haas, France), 5\% (w/w), talc (Luzenac val Chisone SPA, Italy), 4\% (w/w), magnesium stearate (Faci SPA, Italy), 1\% (w/w). Drug, MCC and half of the quantities of talc and magnesium stearate were used intragranulary. The rest of talc and magnesium stearate and the whole quantity of Amberlite were admixed extragranulary to the milled and sieved dry granulate. Milling and sieving of the compacts were performed on the Quadro-Comil U20 machine (Quadro, Canada) using a 1.5-mm sieve. Each powder type was characterised with a particle size distribution based on a sieve analysis (Alpine 200LS-N, Hosokawa, Germany) using the following sieve ranges: $0-0.045 \mathrm{~mm}\left(d_{1}\right), 0.045-$ $0.071 \mathrm{~mm}\left(d_{2}\right), 0.071-0.125 \mathrm{~mm}\left(d_{3}\right), 0.125-0.25 \mathrm{~mm}\left(d_{4}\right), 0.25-0.5 \mathrm{~mm}\left(d_{5}\right), 0.5-$ $0.71 \mathrm{~mm}\left(d_{6}\right), 0.71-1.0 \mathrm{~mm}\left(d_{7}\right)$, and $1.0-1.25 \mathrm{~mm}\left(d_{8}\right)$. Each powder type was characterised by the proportion of particles belonging to each particle size group $\left(w_{i}\right)$. All powder mixtures were compressed into tablets on a Killian T300/40 (IMA, Germany) rotary tablet press equipped with round, concave punches $(\Phi=13 \mathrm{~mm}, \mathrm{R}=26 \mathrm{~mm})$ using different combinations of parameters settings: the main compression force, the pre-compression force and the tableting speed (Table 2). The tablet mass was $0.550 \mathrm{~g}$. Due to the high weight fraction of M-112 in the tablet formulation, the particle size distribution of mixture Direct closely resembles the particle distribution of the plain drug (M-112). Approximately $85 \%$ of the particles in Direct are $<0.071 \mathrm{~mm}$, the compactibilitiy slope is $1.63 \cdot 10^{-2} \pm 9.60 \cdot 10^{-4}$, and the crushing strength of tablets made of pure M112 is $98.37 \mathrm{~N} \pm 5.09 \mathrm{~N}$. Detailed analysis of physical properties was published recently in (Zupančič-Božič et al., 2008). 
[Table 2 about here.]

Nine powder mixtures (Direct - 1 sample, Slugging - 4 samples and Roller 4 samples) were compressed at different combinations of parameters settings during the tableting (process parameter combinations 1.-8.). Mixture Direct was compressed also using process parameter combinations 9. - 12. We produced 76 types of tablets, and a sample of 10 tablets was evaluated from each tablet type.

The tablet types were evaluated in terms of a capping coefficient (CC) during the tablet crushing strength testing, and standard deviations of crushing strength $\left(\sigma_{F}\right)$ and mass $\left(\sigma_{m}\right)$. The tablet was considered to have a capping tendency if the upper part of the tablet completely fell apart from the tablet body during crushing strength testing or if typical relief (a significant step form) appeared on the fractured surface of the tablet, which would indicate that there is a large probability that the tablet would break later during the subsequent steps in production (Zupančič-Božič et al., 2008). The CC was calculated as a fraction of the tablets with a capping tendency compared to the whole tested number of tablets.

The quality description factors of each tablet type were analysed in accordance to the following experimental values: compression parameters settings (the main compression force $(F)$, the pre-compression force $(f)$, the tableting speed $(v))$ and powder mixture parameter the median of the particle size distribution $\left(m_{p}\right)$ of the powder. The median of the particle size distribution represents a statistically calculated descriptor of powder type based on particle size distribution of the powders involved in the study.

\subsection{Principal component analysis}

The dimensionality of the problem, can be identified using principal component analysis (PCA) (Jackson, 1991). PCA calculates linear combinations of regressors, called the principal components, in such a way that the components are linearly independent. The variance of each component indicates its importance. The covariance matrix is calculated from the measured data. Let $\boldsymbol{X}$ denote the matrix of the measurements where each column represents one measured quantity and each row represents one time slice of all the measurements. Thus, the covariance matrix $\boldsymbol{C}$ is calculated as

$$
\boldsymbol{C}=\boldsymbol{X}^{T} \boldsymbol{X}
$$

where the diagonal values represent the variances of the measurements (the regressors). The singular values $(\sigma)$ of the matrix $\boldsymbol{X}$ are equal to the eigenvalues of the covariance matrix $\boldsymbol{C}$ and the corresponding eigenvectors of the matrix $\boldsymbol{C}$ form the transformation matrix $\boldsymbol{T}$, each eigenvector representing 
one column, such that the principal components $\boldsymbol{P}$ are calculated as

$$
P=X T
$$

An analysis of each component singular value reveals which components can be neglected without any significant loss of information; generally, it is possible to omit the components whose sum of singular values share in the sum of all singular values is smaller than or equal to the share of measurement-noise in the measurements.

\subsection{Fuzzy model}

Fuzzy models are often used for modelling of non-linear relations (Oblak and Škrjanc, 2006; Oblak et al., 2007; Blažič and Škrjanc, 2007; Logar et al., 2008; Belič et al., 2003); however, their use in pharmacy remains limited. In the present study a Takagi-Sugeno-type fuzzy model was used (Sugeno and Takagi, 1983). The model consists of if-then logical statements that represent the partial relations between the input and the output variables of the model. A logical statement consists of a premise or an if-part that defines a region of input space, and of consequence that is, in Takagi-Sugeno type, an arbitrary function of the input variables $\left(y_{i}\right)$. For simplicity of interpretation, however, the output functions are normally linear functions of the input variables. Thus, the Takagi-Sugeno model of a system with 2 inputs $x_{1}$ and $x_{2}$, where each input is divided between two fuzzy sets, $x_{1} \in X_{11}, X_{12}$ and $x_{2} \in X_{21}, X_{22}$, and with outputs $y_{i}$ that are linear functions of input variables with coefficients $k_{i j}$ would look like:

$$
\begin{aligned}
& \operatorname{IF}\left(x_{1} \in X_{11}\right) \cap\left(x_{2} \in X_{21}\right) \operatorname{THEN}\left(y_{1}=k_{11} x_{1}+k_{12} x_{2}+n_{1}\right) \\
& \operatorname{IF}\left(x_{1} \in X_{11}\right) \cap\left(x_{2} \in X_{22}\right) \operatorname{THEN}\left(y_{2}=k_{21} x_{1}+k_{22} x_{2}+n_{2}\right) \\
& \operatorname{IF}\left(x_{1} \in X_{12}\right) \cap\left(x_{2} \in X_{21}\right) \operatorname{THEN}\left(y_{3}=k_{31} x_{1}+k_{32} x_{2}+n_{3}\right) \\
& \operatorname{IF}\left(x_{1} \in X_{12}\right) \cap\left(x_{2} \in X_{22}\right) \operatorname{THEN}\left(y_{4}=k_{41} x_{1}+k_{42} x_{2}+n_{4}\right)
\end{aligned}
$$

Each logical statement defines a region of input space and the corresponding output function. Each input variable is first fuzzified, by calculating the memberships of the fuzzy sets that describe the particular input variable. Next, the output of each statement is calculated, and then the outputs are aggregated by calculating the weighted sum of the outputs, the weights being the corresponding results of the premises.

$$
y=\frac{\mu_{1} y_{1}+\mu_{2} y_{2}+\mu_{3} y_{3}+\mu_{4} y_{4}}{\mu_{1}+\mu_{2}+\mu_{3}+\mu_{4}}
$$


Where $\mu_{i}$ represents the result of a premise statement.

$$
\begin{aligned}
& \mu_{1}=\left(x_{1} \in X_{11}\right) \cap\left(x_{2} \in X_{21}\right)=\min \left(x_{1} \in X_{11}, x_{2} \in X_{21}\right) \\
& \mu_{2}=\left(x_{1} \in X_{11}\right) \cap\left(x_{2} \in X_{22}\right)=\min \left(x_{1} \in X_{11}, x_{2} \in X_{22}\right) \\
& \mu_{3}=\left(x_{1} \in X_{12}\right) \cap\left(x_{2} \in X_{21}\right)=\min \left(x_{1} \in X_{12}, x_{2} \in X_{21}\right) \\
& \mu_{4}=\left(x_{1} \in X_{12}\right) \cap\left(x_{2} \in X_{22}\right)=\min \left(x_{1} \in X_{12}, x_{2} \in X_{22}\right)
\end{aligned}
$$

\subsection{Optimisation}

To find the optimal setting for the tableting machine with respect to the powders' mechanical properties, the inputs to the model, representing the powders' properties, must be fixed to the values of the current batch, while the tableting machine's settings can be freely changed within the limits of the machine and the tableting process. As the model represents the effects of the powder characteristics and the tableting machine's settings on the tablet quality factors, it is possible to find the tableting machine settings that result in the minimal values of CC, $\sigma_{F}$, and $\sigma_{m}$. The training and simulation of the models as well as the optimisation of the settings were performed in MATLAB (The MathWorks, Natick, MA, USA) (Mathworks, 1998). To find the optimal setting with respect to the tablet quality, a simplex optimisation method (Fletcher, 1969) that was implemented in MATLAB's fminsearch function was used. The tablet quality was described with the following criterion function $(\mathrm{J})$

$$
J=C C+\frac{\sigma_{F}}{40}+\frac{\sigma_{m}}{0.04}+\frac{10000}{v} .
$$

In the criterion function, tableting speed was included, in order to to take the tableting efficiency into account as well. The constants of the criterion function were set to values that cause balanced influence of all the quality measures on the criterion by scaling all the variables on the interval $[0,1]$.

\section{Results}

\subsection{Identification of the data's dimensionality}

Using a PCA on all the input data (all the data without CC, $\sigma_{F}$, and $\sigma_{m}$ ) showed that there are two significant components in the data. Since the measurement noise could not have been estimated, the significance of principal components was set to $95 \%$ of the sum of all singular values of the measurements. The sum of singular values of the first two principal components was 
already larger than $95 \%$ of the sum of all singular values, which means that all the input data could be reduced to two principal components with only $5 \%$ loss of information in the measured data, indicating that the dimensionality of the problem is most likely 2. However, it must be stated that the PCA can be used for the identification of linearly independent variables, and if the relationship between the variables is non-linear, this can result in an underestimation of the dimensionality. In the first step we used the two most important principal components as an input to the model. The model described the measured data well ( $\mathrm{R}^{2}$ above 0.8 ), however, it was not possible to uniquely calculate process variables back from the principal components. Hence, the choice of principal components as the model inputs is not suitable for the process optimisation purposes. Therefore, a different approach for reducing the input data space had to be chosen. First, we condensed the particle size distribution data by calculating the median of the particle size distribution of the powders. The median $m_{p}$ was calculated as

$$
m_{p}=\frac{\sum_{i=1}^{8} d_{i} w_{i} m_{i}}{\sum_{i=1}^{8} w_{i} d_{i}} .
$$

In equation (8) $d_{i}$ represents the particle size range, $w_{i}$ is the portion of the particles within the particle size range, and $m_{i}$ is the median of the particle size range. The median of the particle size distribution of powders from Slugging group are between $0.0657 \mathrm{~mm}-0.0541 \mathrm{~mm}$, in Roller group the values of $m_{p}$ are between $0.0632 \mathrm{~mm}-0.0557 \mathrm{~mm}$, while the value for the Direct powder type is much lower: $0.0028 \mathrm{~mm}$. The distribution of particle sizes is presented in Figure 1).

[Figure 1 about here.]

Next, we checked what combination of the measured quantities would represent a suitable input for the model. For each quality factor a separate model was designed. The parameters of each model were first identified with all possible inputs: the median of the particle size distribution of the powder $\left(m_{p}\right)$, the main compression force $(F)$, the pre-compression force $(f)$, and the tableting speed $(v)$. In the next step, one of the inputs was removed and the model parameters were identified again. The removed parameter that caused the smallest drop or highest elevation of the $\mathrm{R}^{2}$ value was permanently removed as a suitable input to the model. The elimination proces was continued until, any further removal of the inputs caused a significant drop of the $\mathrm{R}^{2}$ value. The following inputs were identified. The model for the CC prediction needs: the median of the particle size distribution of the powder $\left(m_{p}\right)$, and the main compression force $(F)$. The model for the $\sigma_{F}$ needs the median of the particle size distribution of the powder $\left(m_{p}\right)$, the main compression force $(F)$, and pre-compression force $(f)$ as inputs. While the model for the $\sigma_{m}$ needs the 
median of the particle size distribution of the powder $\left(m_{p}\right)$, the main compression force $(F)$, the pre-compression force $(f)$, and the tableting speed $(v)$ as inputs.

\subsection{Model prediction}

For validation purposes a variant of the leave-one-out procedure was used; however, for the final results, all the available data were used for the identification. Initially, each input to the model was described with two fuzzy sets and their trapezoidal membership functions were identified in the model identification process.

\subsubsection{Fuzzy model of $C C$}

The identified model for CC described the input-output relation with two fuzzy sets for each linguistic variable on the input, resulting in four logical statements. The linguistic variable, median of the particle size distribution was described by two sets that were named, fine and coarse, while the main compression force was described with the sets that were named high and low. The naming of the sets was chosen according to the interpretation of the identified membership functions. The membership functions for the fuzzy sets

were identified with the MATLAB's function anfis, and are presented in Figure 2 .

[Figure 2 about here.]

The logical statements describing the model are as follows:

$$
\begin{gathered}
\operatorname{IF}\left(m_{p} \in \text { fine }\right) \cap(F \in \text { low }) \operatorname{THEN}\left(C C_{1}=-0.001 m_{p}+0.042 F-0.296\right) \\
\operatorname{IF}\left(m_{p} \in \text { fine }\right) \cap(F \in \text { high }) \operatorname{THEN}\left(C C_{2}=-0.011 m_{p}-0.225 F-3.75\right) \\
\operatorname{IF}\left(m_{p} \in \text { coarse }\right) \cap(F \in \text { low }) \operatorname{THEN}\left(C C_{3}=-0.067 m_{p}+0.003 F\right) \\
\operatorname{IF}\left(m_{p} \in \text { coarse }\right) \cap(F \in \text { high }) \operatorname{THEN}\left(C C_{4}=-11.81 m_{p}-0.031 F+1.4796\right)
\end{gathered}
$$

while the output of the fuzzy model is:

$$
C C=\frac{\mu_{1} C C_{1}+\mu_{2} C C_{2}+\mu_{3} C C_{3}+\mu_{4} C C_{4}}{\mu_{1}+\mu_{2}+\mu_{3}+\mu_{4}}
$$

where $\mu_{i}$ is a value of the fuzzy intersection for the premise of each statement. The resulting relation for the $\mathrm{CC}$ identified with the fuzzy model is presented in Figure 3.

[Figure 3 about here.] 
The quality of the fuzzy identification process was assessed by a calculation of $\mathrm{R}^{2}$. The coefficient $\mathrm{R}^{2}$ for the model was 0.7 (Figure 3 ). The estimated reliability of the model for the CC was \pm 0.3 .

\subsubsection{Fuzzy model of $\sigma_{F}$}

The model for prediction of $\sigma_{F}$ has one additional input with respect to the model of CC. Identification of the model resulted in almost identical placement of the two membership functions for the linguistic variables the median of the particle size distribution and main compression force as in the case of CC. The identified membership functions of the linguistic variable pre-compression force are shown in Figure 4.

[Figure 4 about here.]

Similar to the fuzzy sets for the main compression force, the two fuzzy sets were named high and low. The logical and the output statements are similar to the statements of the CC model, except that there is one more input variable which elevates the number of logical statements to eight, since we have three input variables and each of them can be a member of the two sets. The output statement is then composed of eight terms; one term for each logical statement. The resulting relation for the $\sigma_{F}$ identified with the fuzzy model is presented in Figure 5.

[Figure 5 about here.]

The quality of the fuzzy identification process was assessed by a calculation of $\mathrm{R}^{2}$. The coefficient $\mathrm{R}^{2}$ for the model was 0.7 (Figure 5). The estimated reliability of the model for the $\sigma_{F}$ prediction was $\pm 10 \mathrm{~N}$.

\subsubsection{Fuzzy model of $\sigma_{m}$}

Tableting speed $(v)$ had to be added as an additional input with respect to the model of $\sigma_{F}$. Identification of the model resulted in almost identical membership functions for the linguistic variables: median of the particle size distribution, main compression force, and pre-compression force as for models of $\mathrm{CC}$ and $\sigma_{F}$. The identified membership functions of the linguistic variable tableting speed are shown in Figure 6.

[Figure 6 about here.]

The two fuzzy sets of the variable tableting speed were named high and low. The logical and the output statements are similar to the statements of the $\mathrm{CC}$ model, except that there are two more input variables which elevates the 
number of logical statements to 16 , since we have four input variables and each of them can be a member of the two sets. The output statement is then composed of 16 terms; one term for each logical statement. The resulting relation for the $\sigma_{m}$ identified with the fuzzy model is presented in Figure 7.

[Figure 7 about here.]

The quality of the fuzzy identification process was assessed by a calculation of $\mathrm{R}^{2}$. The coefficient $\mathrm{R}^{2}$ for the model was 0.7 (Figure 7 ). The estimated reliability of the model for the $\sigma_{m}$ was $\pm 0.005 \mathrm{~g}$.

\subsection{Optimisation}

The optimisation was performed only for the Direct system, since both dry granulation systems have resulted in already sufficient tablet quality for all the selected machine settings. When optimising the tableting machine settings, the mechanical properties of the powder are a-priori known, therefore, only a part

of the modelled surfaces (Figures 3,5 and 7) at the value of $m_{p}$ that describes the median of the particle size distribution of the actual powder is relevant. The capping coefficient (CC) becomes a function of the main compression force (Figure 8).

[Figure 8 about here.]

The standard deviation of crushing strength $\left(\sigma_{F}\right)$ becomes a function of the main compression force $(F)$ and the pre-compression force $(f)$ (Figure 9).

[Figure 9 about here.]

The standard deviation of tablet mass $\left(\sigma_{m}\right)$ becomes a function of the main compression force $(F)$, the pre-compression force $(f)$, and the tableting speed $(v)$, however, since best economical effect is achieved at the top tableting speed, only the relation at $100,000 \mathrm{tbl} / \mathrm{h}$ is relevant (Figure 10).

[Figure 10 about here.]

In Figures 8 to 10 the areas with optimal settings for the tableting machine for the Direct system are located in two regions. The first-one is around $F=7 \mathrm{kN}$ and $f=5.5 \mathrm{kN}$, and the second-one is around $F=15 \mathrm{kN}$ and $f=7 \mathrm{kN}$. In both cases tableting speed is set to $100,000 \mathrm{tbl} / \mathrm{h}$. 


\section{Discussion}

The most important issue during modelling is the model validation. The validation of models that were built exclusively on data (identification) is usually done by testing the model's predictive power. In our case a variant of the leave-one-out procedure was used. Thus, for each identification-validation cycle, 61 randomly chosen tablet types out of 76 were used for the identification, while the model was validated on the remaining 15 tablet types. The model validation is discussed in detail in (Belič et al., 2009).

The model indicated two areas of machine parameters settings that can lead to optimal tablet quality. The procedure for optimal setting of the machine with respect to the powder batch properties must continue by testing some settings in the identified optimal areas on the tableting machine. The area with low main and pre- compression forces may have some negative effects on the absolute value of the crushing strength, therefore, the area with higher values of the main and pre-compression forces should be tested first. The data collected from the tests must be included to the training data set, since it will add valuable information on the identified relation and the model will be upgraded to produce more precise predictions. Thus, with every new powder batch, the model will become more reliable.

Various information on process characteristics can be extracted from the model as well. The identified membership functions (Figures 2, 4,6) show that fuzzification separates the median of the particle size distribution such that mixtures with the values below $0.02 \mathrm{~mm}$ are considered as fine mixtures that might have poor compressibility and flow characteristics, while the mixtures with the values above $0.05 \mathrm{~mm}$ are considered as coarse mixtures with relatively good compressibility and flow characteristics. The main compression forces below $13 \mathrm{kN}$ are considered as low forces, while forces above $18 \mathrm{kN}$ are considered as high forces. Therefore, it can be stated that the powder mixture, used in the study, shows different compression characteristics under conditions of low or high main compression forces, suggesting at least two different dominant mechanisms of particle adhesion at compression. The pre-compression force is considered to be low under $7.5 \mathrm{kN}$, while it is considered high above $8.8 \mathrm{kN}$, again suggesting at least two different dominant mechanisms at precompression. The tableting speed is also divided into two regions: low, which is below 50,000 tbl/h, and high, which is above $80,000 \mathrm{tbl} / \mathrm{h}$. The results in Figure 7 in combination with such division of the tableting speed space suggest that flow characteristics of the powder and the tableting machine characteristics may start causing problems with accurate dosing of the powder to the tableting dye at high tableting speeds. 


\section{Conclusion}

Fuzzy models are not very often used in the field of pharmaceutical technology; however, they have some significant advantages over other model types. Most importantly, it is possible to use other knowledge than just the measured data for their identification, which reduces the need for large quantities of data when identifying non-linear relations. The fuzzy model can be built on the basis of piece-wise linear models that are often used in pharmaceutical sciences. Thus, we also obtain better model transparency, which is very important for the understanding of complex non-linear relations.

The identified input-output relation is specific to the tableting machine, the equipment used in the study, as well as the powder mixture and cannot be generalised to all tableting machines of the same model or all powder mixtures. However, the presented procedure of model identification is generally applicable to all tableting procedures and machines.

The model represents valuable information for the operator, about optimal ranges of machine settings with respect to the physical properties of the powder. Using the model is also more cost effective than a manual trial-and-error approach. Optimisation of the tableting machine's settings by trial and error produces a relatively large number of faulty tablets and is very time consuming, especially when the starting settings of the machine were poorly guessed; furthermore, the procedure has to be repeated for each new powder batch. In an industrial environment with a PAT system implemented the development of the model would have to be divided into two stages. First, the data generation for building the model would be organised as a dedicated experiment that should cover the area of interest described by machine's setting parameters and the significant properties of the powder. For the modelling, the machine's settings must be systematically chosen to cover the whole area of interesting values and tested for several batches. Next, the model would be validated and further developed with data from large-scale production. Production monitoring data typically contributes relatively dense data sets from the near-optimal areas of the experimental space that were not included in the experimental plan at the beginning. The experiments for modelling purposes are more expensive than the ones for the optimisation with trial-and-error; however, the model can be used for the prediction of optimal settings for new batches, which substantially shortens the time for optimising the machine settings with respect to a new batch and reduces the number of faulty tablets. The model, developed in the present study is suitable for implementation into the PAT concept and can become an important part of a QbD approach. 


\section{References}

Belič, A., Grabnar, I., Karba, R., Mrhar, A., 2003. Pathways of paracetamol absorption from layered excipient suppositories: artificial intelligence approach. Eur. J. Drug Metab. Pharmacokinet. 28 (1), 31-40.

Belič, A., Škrjanc, I., Zupančič-Božič, D., Karba, R., Vrečer, F., 2009. Minimisation of the capping tendency by tableting process optimisation with the application of artificial neural networks and fuzzy models. Eur. J. Pharm. Biopharm., doi:10.1016/j.ejpb.2009.05.005.

Blažič, S., Škrjanc, I., 2007. Design and stability analysis of fuzzy model-based predictive control - a case study. J. Intell. Robot. Syst. 49 (3), 279-292.

Busignies, V., Leclerc, B., Porion, P., Evesque, P., Couarraze, G., Tchoreloff, P., 2006. Compaction behaviour and new predictive approach to the compressibility of binary mixtures of pharmaceutical excipients. Eur. J. Pharm. Biopharm. 64, 66-74.

Carstensen, J., 1996. Modeling and Data Treatment in the Pharmaceutical Sciences. Technomic Publishing Company, Lancaster.

Fletcher, R., 1969. Optimization. Academic Press, London.

Frake, P., Greenhalgh, D., Grierson, S. M., Hempenstall, J. M., Rud, D. R., 1997. Process control and end-point determination of fluid bed granulation by application of near infra-red spectroscopy. Int. J. Pharm. 151, 75-80.

Gohel, M. C., Jogani, P., 2002. Functionality testing of a multifunctional directly compressible adjuvant containing lactose, polyvinylpyrrolidone and croscarmellose sodium. Pharm. Tech. 3, 64-82.

Ilkka, J., Paronen, P., 1993. Prediction of the compression behaviour of powder mixtures by heckel equation. Int. J. Pharm. 94, 181-187.

Informa, 2007. PAT - Quality by design and process improvement. Informa, Amsterdam.

Jackson, J. E., 1991. A User Guide to Principal Components. John Wiley \& Sons, inc., New York.

Li, Q., Rudolph, V., Weigl, B., Earl, A., 2004. Interparticle van der waals force in powder flowability and compactability. Int. J. Pharm. 280, 77-93.

Logar, V., Škrjanc, I., Belič, A., Karba, R., Brežan, S., Koritnik, B., Zidar, J., 2008. Gripping-force identification using eeg and phase demodulation approach. Neurosci. Res. 60 (4), 389-396.

Luangtana-Anan, M., Fell, J. T., 1990. Bonding mechanisms in tabletting. Int. J. Pharm. 60, 197-202.

Mathworks, 1998. Using Matlab version 5. The Mathworks Inc., Natick.

Nyström, C., Alderborn, G., Duberg, M., Karerhill, P., 1993. Bonding surface area and bonding mechanism - two important factors for the understanding of powder compactability. Drug. Dev. Ind. Pharm. 19, 2143-2196.

Oblak, S., Škrjanc, I., 2006. A comparison of fuzzy and cpwl approximations in the continuous-time nonlinear model-predictive control of time-delayed wiener-type systems. J. Intell. Robot. Syst. 47 (2), 125-127.

Oblak, S., Škrjanc, I., Blažič, S., 2007. Fault detection for nonlinear systems with 
uncertain parameters based on the interval fuzzy model. Eng. Appl. Artif. Intell. 20 (4), 503-510.

Parrott, E. L., 1990. Pharmaceutical dosage forms. Tablets. Marcel Dekker Inc., New York, Ch. Compression, pp. 201-243.

Picker, K., 2001. Time dependence of elastic recovery for characterization of tableting materials. Pharmaceut. Dev. Tech. 6 (1), 61-70.

Rios, M., 2006. Developments in powder flow testing. Pharm. Technol. Feb., 38-49.

Sebhatu, T., Ahlneck, C., Alderborn, G., 1997. The effect of moisture content on the compressional and bond-formation properties of amorphous lactose particles. Int. J. Pharm. 146, 101-114.

Sonnergaard, J. M., 2006. Quantification of the compactibility of pharmaceutical powders. Eur. J. Pharm. Bioph. 63, 270-277.

Sorensen, A. H., Sonnergaard, J. M., Hovgard, L., 2006. Bulk characterisation of pharmaceutical powders by low-pressure compression ii: Effect of method settings and particle size. Pharm. Dev. Technol. 11, 235-241.

Sucker, H., 1982. Test methods for granulates. Pharm. Ind. 44 (3), 312-316.

Sugeno, M., Takagi, T., 1983. Multi-dimensional fuzzy reasoning. Fuzzy Sets Syst. 9, 313-325.

Wu, C. Y., Ruddy, O. M., Bentham, A. C., Hancock, B. C., Best, S. M., Elliott, J. A., 2005. Modelling the mechanical behaviour of pharmaceutical powders during compaction. Powder Tech. 152, 107-117.

Zhang, Y., Law, Y., Chakrabarti, S., 2003. Physical properties and compact analysis of commonly used direct compression binders. AAPS PharmSciTech 4 (4), art. 62.

Zupančič-Božič, D., Dreu, R., Vrečer, F., 2008. Influence of dry granulation on compactibility and capping tendency of macrolide antibiotic formulation. Int. J. Pharm. 357 (1-2), 44-54. 


\section{List of Figures}

1 Boxplot of the particle size distributions for the mixtures used in the study. The middle line in the box represents the median, the box represents the upper and the lower quartile of the particle size distribution, and the whiskers represent the total data extent.

2 Membership functions for the linguistic variables: a) the median of the particle size distribution $\left(m_{p}\right)$, b) the main compression force $(F)$

3 Non-linear relation between the main compression force $(F)$, the median of the particle size distribution $\left(m_{p}\right)$ and the capping coefficient $(\mathrm{CC})$. The circles represent the measurements; the surface represents the prediction of the fuzzy model.

4 Membership functions for the linguistic variable precompression force $(f)$

$5 \quad$ Standard deviation of crushing strength $\left(\sigma_{F}\right)$ with respect to the median of the particle size distribution $\left(m_{p}\right)$, the main compression force $(F)$, and the pre-compression force $(f)$

6 Membership functions for the linguistic variable tableting speed $(v)$

$7 \quad$ Standard deviation of mass $\sigma_{m}$ with respect to the median of the particle size distribution $\left(m_{p}\right)$, the main compression force $(F)$, the pre-compression force $(f)$, and the tableting speed $(v)$. Only the settings that were experimentally tested are shown.

8 CC with respect to main compression force $(F)$ for the Direct powder. Circles indicate settings that yield optimal-quality tablets.

$9 \quad$ Standard deviation of crushing strength $\sigma_{F}$ with respect to main compression force $(F)$ and pre-compression force for the Direct powder as identified with the model. White areas indicate settings that yield optimal-quality tablets. 
10 Standard deviation of mass $\left(\sigma_{m}\right)$ with respect to the main compression force $(F)$, the pre-compression force $(f)$, at maximum tableting speed $v=100,000 \mathrm{tbl} / \mathrm{h}$ for the Direct powder. White areas indicate settings that yield optimal-quality tablets. 


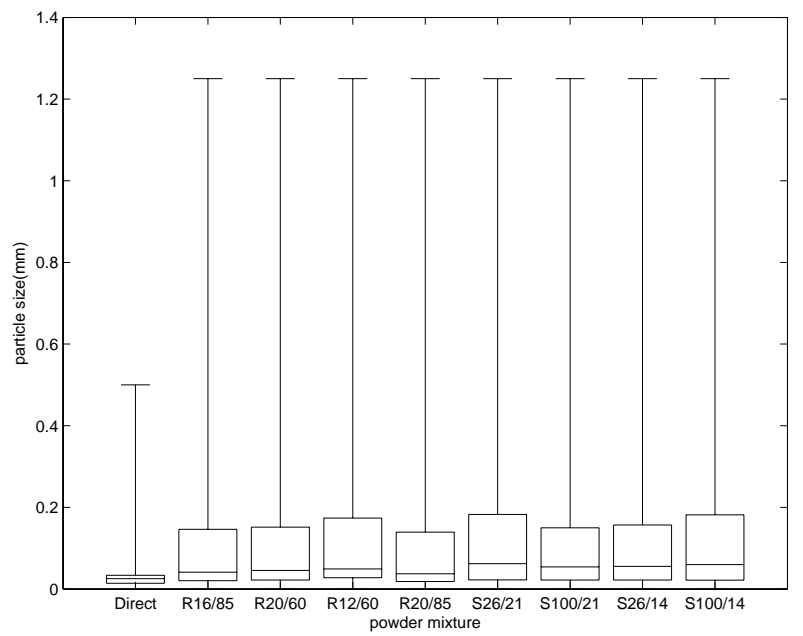

Figure 1. Boxplot of the particle size distributions for the mixtures used in the study. The middle line in the box represents the median, the box represents the upper and the lower quartile of the particle size distribution, and the whiskers represent the total data extent. 

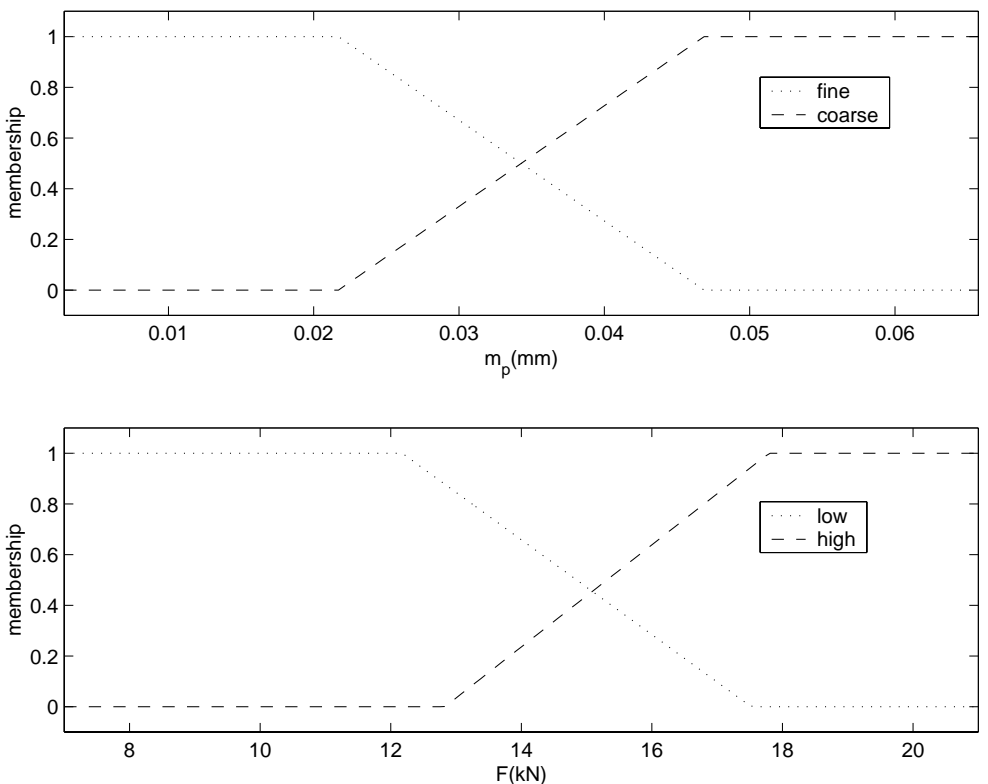

Figure 2. Membership functions for the linguistic variables: a) the median of the particle size distribution $\left(m_{p}\right)$, b) the main compression force $(F)$ 


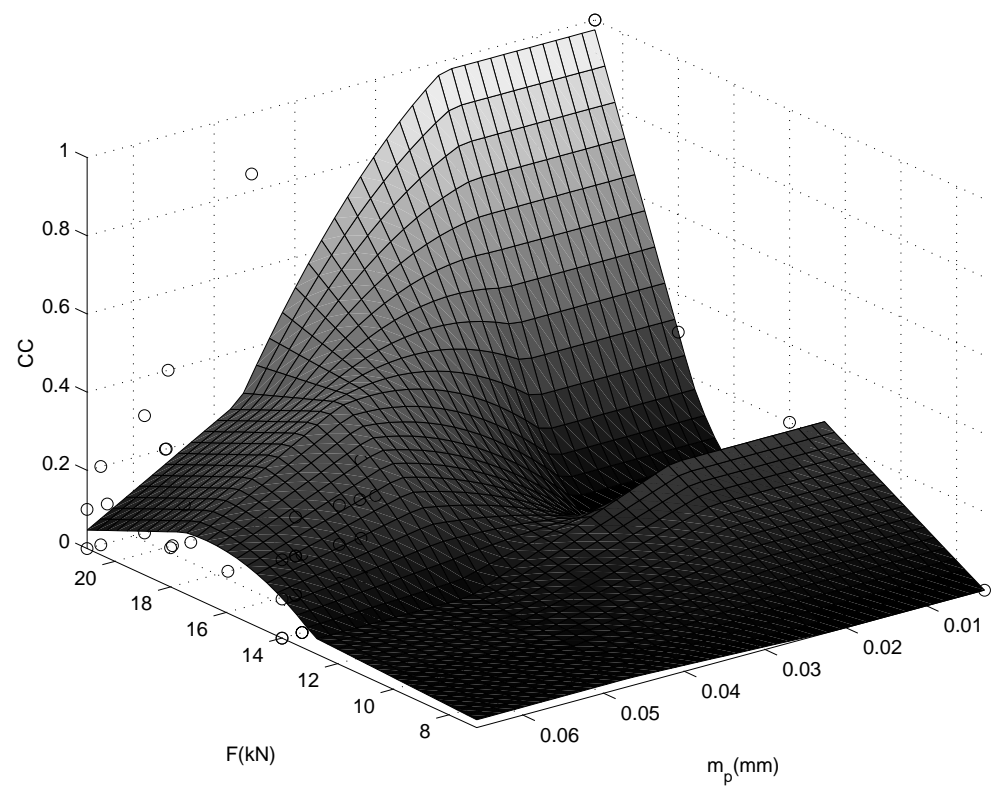

Figure 3. Non-linear relation between the main compression force $(F)$, the median of the particle size distribution $\left(m_{p}\right)$ and the capping coefficient $(\mathrm{CC})$. The circles represent the measurements; the surface represents the prediction of the fuzzy model. 


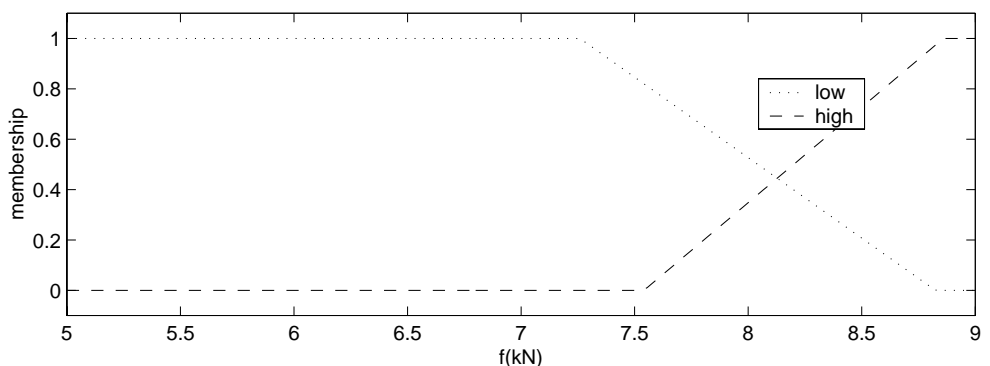

Figure 4. Membership functions for the linguistic variable pre-compression force $(f)$ 
$f=5 \mathrm{kN}$

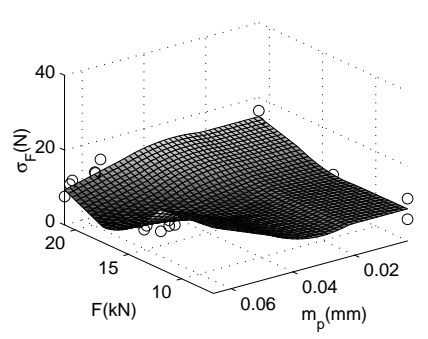

$f=7.5 \mathrm{kN}$

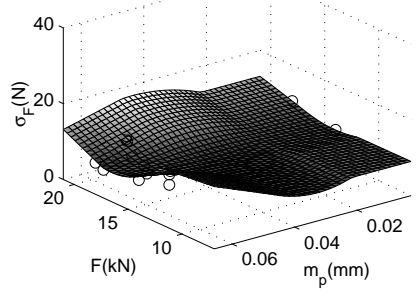

$f=9 \mathrm{kN}$

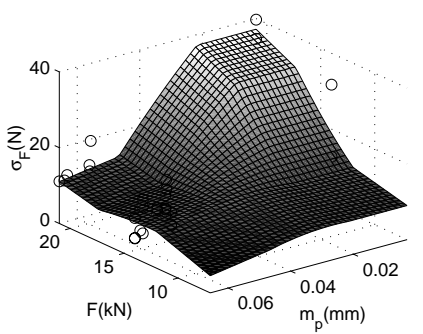

Figure 5. Standard deviation of crushing strength $\left(\sigma_{F}\right)$ with respect to the median of the particle size distribution $\left(m_{p}\right)$, the main compression force $(F)$, and the pre-compression force $(f)$ 


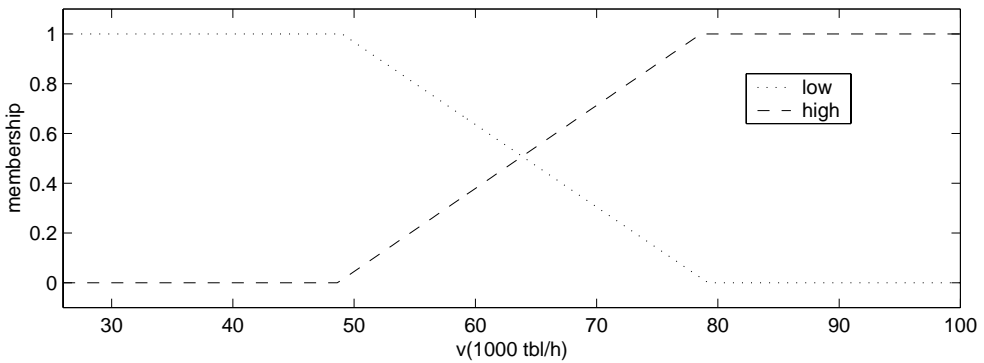

Figure 6. Membership functions for the linguistic variable tableting speed $(v)$ 


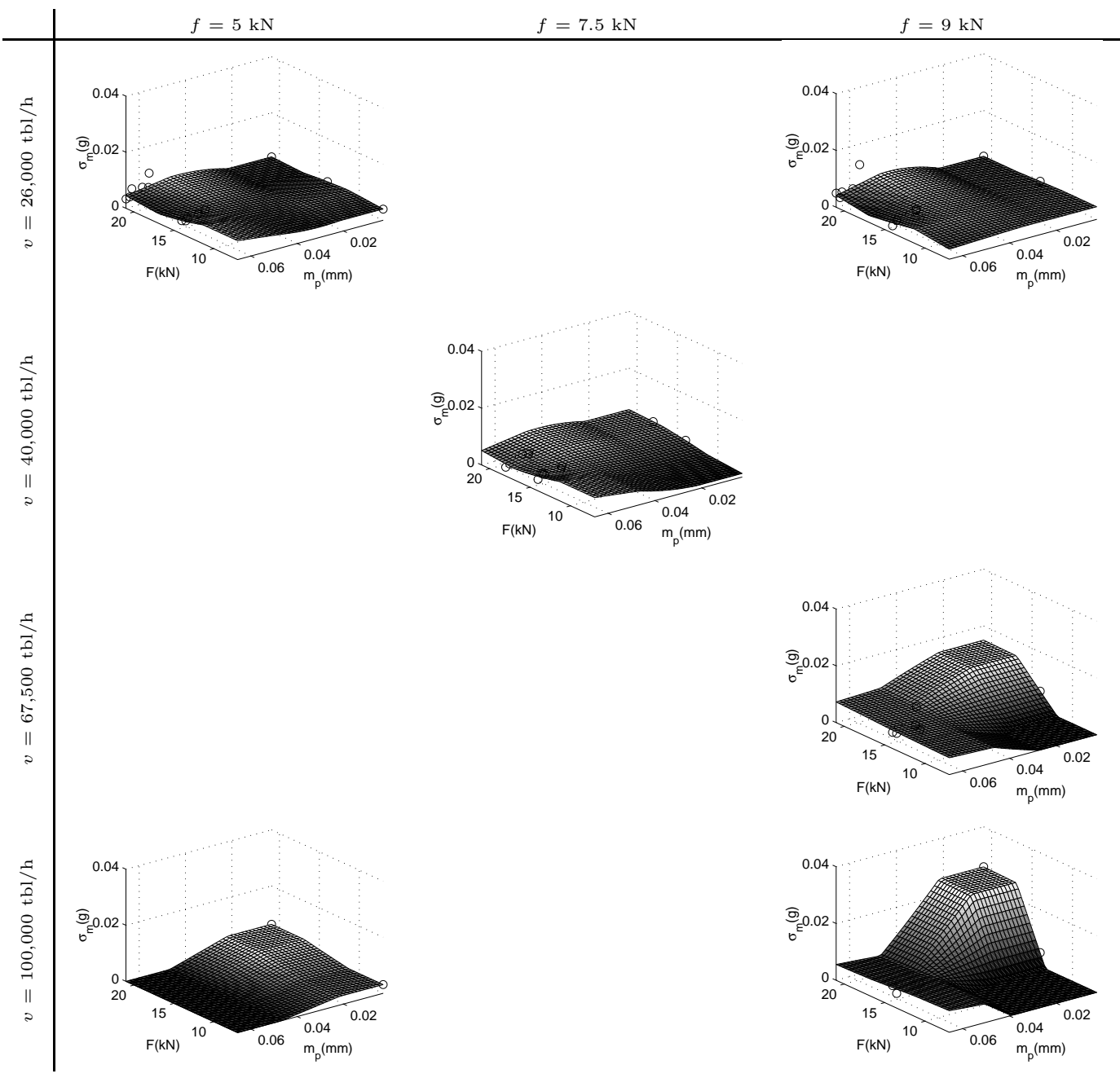

Figure 7. Standard deviation of mass $\sigma_{m}$ with respect to the median of the particle size distribution $\left(m_{p}\right)$, the main compression force $(F)$, the pre-compression force $(f)$, and the tableting speed $(v)$. Only the settings that were experimentally tested are shown. 


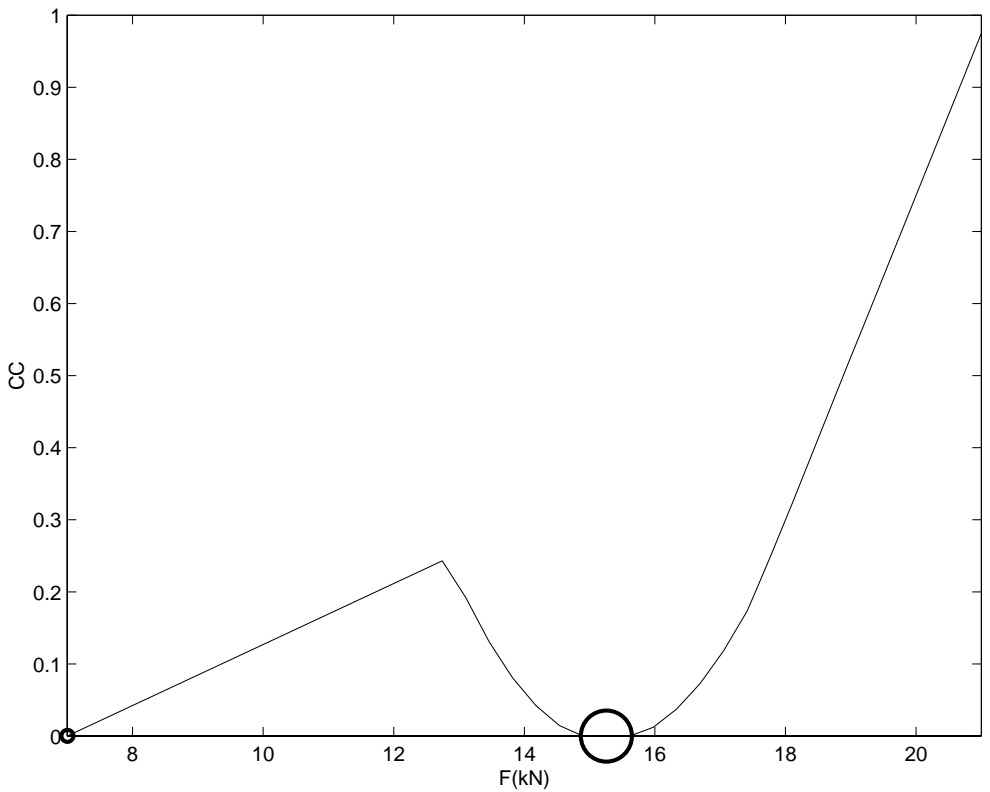

Figure 8. CC with respect to main compression force $(F)$ for the Direct powder. Circles indicate settings that yield optimal-quality tablets. 


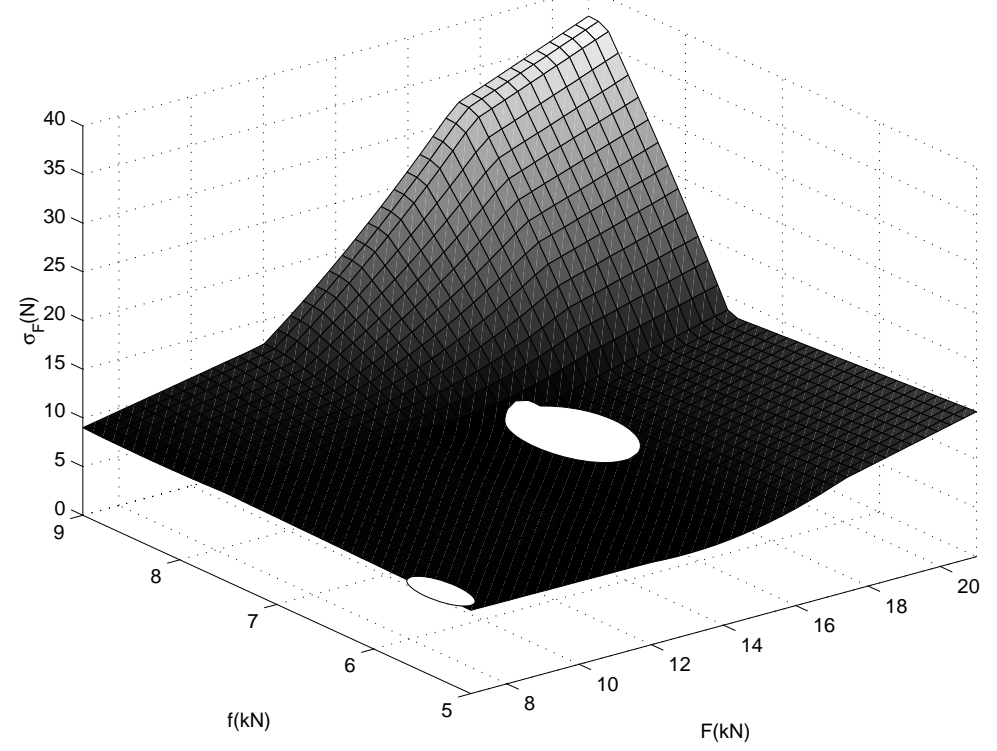

Figure 9. Standard deviation of crushing strength $\sigma_{F}$ with respect to main compression force $(F)$ and pre-compression force for the Direct powder as identified with the model. White areas indicate settings that yield optimal-quality tablets. 


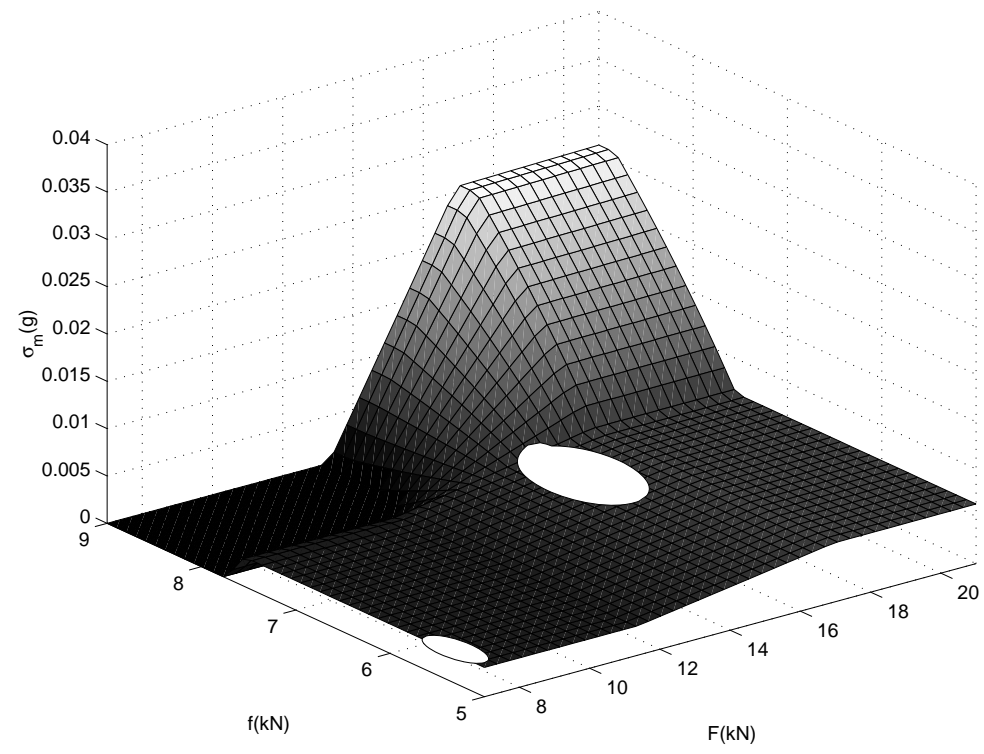

Figure 10. Standard deviation of mass $\left(\sigma_{m}\right)$ with respect to the main compression force $(F)$, the pre-compression force $(f)$, at maximum tableting speed $v=$ $100,000 \mathrm{tbl} / \mathrm{h}$ for the Direct powder. White areas indicate settings that yield optimal-quality tablets. 


\section{List of Tables}

1 Process parameters for dry granulation on a rotary tablet press (Slugging) and on a roller compactor (Roller)

2 Combinations of the process parameters settings 
Table 1

Process parameters for dry granulation on a rotary tablet press (Slugging) and on a roller compactor (Roller)

\begin{tabular}{|c|c|c|c|c|c|}
\hline \multicolumn{3}{|c|}{ Slugging } & \multicolumn{3}{c|}{ Roller } \\
\hline label & $\begin{array}{c}\text { speed } \\
(\mathrm{x} 1000 \mathrm{tbl} / \mathrm{h})\end{array}$ & $\begin{array}{c}\text { compression } \\
\text { force } \\
(\mathrm{kN})\end{array}$ & label & $\begin{array}{c}\text { speed } \\
(\mathrm{x} 1000 \mathrm{tbl} / \mathrm{h})\end{array}$ & $\begin{array}{c}\text { compression } \\
\text { force } \\
(\mathrm{kN})\end{array}$ \\
\hline $\mathrm{S} 26 / 21$ & 26 & 21 & $\mathrm{R} 12 / 60$ & 12 & 60 \\
\hline $\mathrm{S} 100 / 21$ & 100 & 21 & $\mathrm{R} 20 / 60$ & 20 & 60 \\
\hline $\mathrm{S} 26 / 14$ & 26 & 14 & $\mathrm{~S} 16 / 85$ & 16 & 85 \\
\hline $\mathrm{S} 100 / 14$ & 100 & 14 & $\mathrm{~S} 20 / 85$ & 20 & 85 \\
\hline
\end{tabular}


Table 2

Combinations of the process parameters settings

\begin{tabular}{|c|c|c|c|}
\hline $\begin{array}{c}\text { Process } \\
\text { parameters } \\
\text { combination }\end{array}$ & $\begin{array}{c}\text { Main compression } \\
\text { force } \\
(\mathrm{kN})\end{array}$ & $\begin{array}{c}\text { Pre-compression } \\
\text { force } \\
(\mathrm{kN})\end{array}$ & $\begin{array}{c}\text { Tableting speed } \\
(\mathrm{x} 1000 \mathrm{tbl} / \mathrm{h})\end{array}$ \\
\hline 1. & 21 & 5 & 26 \\
\hline 2. & 21 & 9 & 26 \\
\hline 3. & 14 & 9 & 26 \\
\hline 4. & 14 & 5 & 26 \\
\hline 5. & 18 & 7.5 & 40 \\
\hline 6. & 14 & 7.5 & 40 \\
\hline 7. & 14 & 9 & 100 \\
\hline 8. & 14 & 9 & 100 \\
\hline 9. & 21 & 5 & 100 \\
\hline 10. & 21 & 9 & 100 \\
\hline 11. & 7 & 5 & 26 \\
\hline 12. & 7 & 5 & \\
\hline
\end{tabular}

